\title{
Ferramenta gerencial para integração dos serviços de saúde na gestão de riscos de desastres: o caso de Blumenau, SC
}

\author{
Management tool for the integration of health services in disaster risk \\ management: the case of Blumenau, $S C$
}

Sherelee Ribeiro Spindola de Moura', João Marcos Bosi Mendonça de Moura', Rafaela Vieira'

DOI: 10.1590/0103-11042020E211

\begin{abstract}
RESUMO No âmbito dos desastres, os planos de contingência em saúde costumam limitar-se aos aspectos de resposta e emergência. Propõe-se que, em uma perspectiva holística, os municípios formulem planos que contemplem também aspectos de prevenção e redução de riscos. Nesse contexto, objetivou-se desenvolver e aplicar um instrumento técnico para a formulação de um plano de ação dos serviços da saúde voltado à Gestão de Riscos de Desastres (GRD). As bases conceituais apresentadas fundamentaram a elaboração do instrumento levando-se em conta uma gestão integral, integrada e participativa. Programas, projetos, ações, agentes e instrumentos jurídicos são descritos a partir dos diversos processos da GRD do município de Blumenau, Santa Catarina. Os resultados revelaram um diagnóstico das políticas do município. As ações existentes concentram-se em processos de preparação e resposta. O Programa Vigidesastres não se encontra operante. Novas ações também são propostas com o intuito de fortalecer ações de prevenção e redução de riscos. Espera-se que o estudo possa subsidiar políticas públicas que considerem os serviços de saúde sob uma perspectiva integral, reduzindo as vulnerabilidades sociais e promovendo a saúde da população.
\end{abstract}

PALAVRAS-CHAVE Política pública. Desastres naturais. Saúde ambiental. Vigilância em saúde.

\begin{abstract}
Health contingency plans are usually limited to response and emergency aspects in the context of disasters. It is proposed that, in a holistic perspective, the municipalities formulate plans that also include prevention and reduction of risks. Thus, the aim of this study was to develop and apply a technical instrument for the formulation of a health services action plan for Disaster Risk Management (GRD). The conceptual bases were used for the elaboration of the instrument, taking into account integral, integrated, and participative management. Programs, projects, actions, agents and legal instruments are described from the various GRD processes in the municipality of Blumenau, Santa Catarina. The results revealed a diagnosis of municipal policies. The actions are limited to the preparation and response processes and the 'Vigidesastres' Program is not operational. New actions are also proposed to improve public health policies in your prevention and mitigation processes. It is hoped that the study can subsidize public policies that consider health services from an integral and integrated perspective, reducing social vulnerabilities and promoting populational health.
\end{abstract}

KEYWORDS Public Policy. Natural Disasters. Environmental Health. Public Health Surveillance. 


\section{Introdução}

Os riscos de desastres são construídos por meio de uma combinação complexa de aspectos ambientais, políticos e sociais $\mathbf{1}$. Denotam a possibilidade de danos à sociedade, mas também resultam do seu próprio modelo de desenvolvimento ${ }^{2}$. Portanto, a noção de desastre não apenas remonta à ideia de perdas e danos que interrompem de maneira significativa o funcionamento da sociedade, mas que também o próprio evento é construído por ela ${ }^{3}$.

Um olhar voltado ao risco possibilita identificar os fatores que 'anunciam' o desastre e que são responsáveis pela distribuição desigual de seus impactos negativos ${ }^{3}$. Óbitos, doenças e prejuízos aos serviços e estabelecimentos de saúde podem ser produzidos pelos desastres ${ }^{4}$, porém sua magnitude e frequência serão tão altas quanto for a vulnerabilidade local.

A vulnerabilidade pode ser definida como uma predisposição ao dano que seres humanos e seus meios de vida constroem em interação com o meio ambiente ante as ocorrências de eventos naturais ou tecnológicos perigosos ${ }^{3}$. Reconhece-se, portanto, a necessidade de ir além das respostas emergenciais e compreender como a vulnerabilidade se constrói e como é possível reduzi-la.

Após a implementação da Política Nacional de Proteção e Defesa Civil (PNPDEC) ${ }^{5}$, houve expressivo aumento da elaboração de planos de contingência em saúde no Brasil6. São documentos que contemplam aspectos de alerta, assistência, socorro e restabelecimento dos serviços essenciais. Por regra, limitam-se a abordar os processos de preparação, resposta e recuperação (gestão 'reativa'), sem uma reflexão mais aprofundada sobre a construção das vulnerabilidades e do risco.

Isso se traduz na fragilização de uma gestão 'prospectiva' e 'corretiva', isto é, que aborde a prevenção e a redução dos riscos de desastre. No âmbito da saúde, a prevenção e a redução de riscos podem ser fortalecidas por meio dos seus profissionais da atenção primária. Os agentes comunitários da saúde são profissionais com forte relação com a comunidade e alto nível de conhecimento territorial, aspectos que poderiam ser explorados em políticas públicas de redução dos riscos de desastres ${ }^{7}$.

Diante disso, objetivou-se apresentar uma ferramenta gerencial que facilite o processo de construção de um plano de ação do setor saúde na Gestão de Riscos de Desastres (GRD) do município de Blumenau, Santa Catarina (SC). A proposta visa fortalecer uma visão holística, integrada e participativa do processo de GRD. Medidas de prevenção e redução de riscos são consideradas, fazendo proveito do capital social existente dos profissionais da saúde nos mais diversos níveis.

Um instrumento foi desenvolvido para a formulação de um plano de ação que busca articular programas, projetos, ações, agentes e instrumentos jurídicos existentes (e também os não existentes, mas necessários) ao longo dos processos da GRD. $\mathrm{Na}$ pesquisa, aplica-se o instrumento em Blumenau (SC), município com registros frequentes de inundações e movimentos gravitacionais de massa ${ }^{1}$.

\section{Material e métodos}

\section{Área de estudo}

O município de Blumenau (SC) possui população estimada de 357.199 habitantes, Índice de Desenvolvimento Humano Municipal de 0,806 e o $4^{\circ}$ maior Produto Interno Bruto (PIB) do estado. A cidade é um polo nacional importante da indústria têxtil e da tecnologia da informação ${ }^{8}$.

A atenção básica de saúde cobre $96 \%$ do território municipal. Das 66 equipes da Estratégia Saúde da Família (ESF) existentes, 54 atuam em áreas de alta e média suscetibilidade aos riscos de desastre associados à inundação e movimentos de gravitacionais de massa ${ }^{9}$ (figura 1). 
Figura 1. Localização do município de Blumenau, Santa Catarina, e o rio Itajaí-açu, principal curso d'água da bacia hidrográfica do rio Itajaí

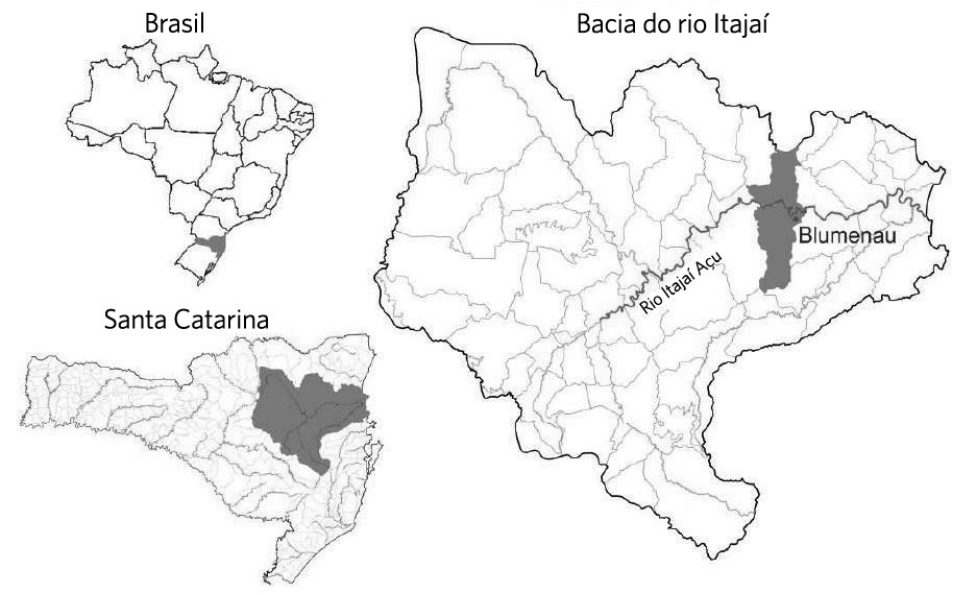

Fonte: Vieira, Schmidt e Moura

Desde os primórdios de sua colonização no século XIX, há registros de intensos desastres associados à inundação do rio Itajaí-Açu ${ }^{10}$. Em novembro de 2008, um acumulado mensal de chuva igual a $1.002 \mathrm{~mm}$ (quantidade $600 \%$ superior à média mensal) desencadeou simultaneamente a ocorrência de inundações, enxurradas e movimentos gravitacionais de massa na região. No Vale do Itajaí, foram 103 mil pessoas afetadas, 5,2 mil habitantes desabrigados, 25 mil desalojados, 2,4 mil feridos ou gravemente feridos e 24 mortos ${ }^{11}$.

As grandes inundações de 1983 e 1984 foram também destaque no noticiário nacional. De 2009 a 2019, mais dez inundações foram registradas no município ${ }^{12}$. Ao longo desses eventos, são registrados danos significativos à saúde da população (depressão, Acidente Vascular Cerebral - AVC, leptospirose, infecções alimentares etc.), bem como prejuízos aos estabelecimentos de saúde ${ }^{11,13}$.

A Diretoria de Ações de Saúde do município participa das reuniões no Grupo de Ações Coordenadas do Plano de Contingência contra Inundações (Grac - Decreto $n^{\circ} 1.879 / 13$ ), que é formado por diversos órgãos, como: Defesa Civil, Polícia Militar e outras secretarias municipais. O Grac atua somente durante ou na iminência de um desastre' ${ }^{\mathbf{1}}$.
Embora haja um plano de contingência específico e vinculado à secretaria municipal de promoção à saúde ${ }^{\mathbf{1 4}}$, observa-se que as ações previstas são de caráter emergencial com ênfase na instalação e operação de abrigos públicos. O Programa Nacional de Vigilância em Saúde Ambiental dos Riscos Decorrentes dos Desastres Naturais (Vigidesastres) é contemplado no plano, contudo, não se encontra em operação?

\section{Bases conceituais}

A PNPDEC - Lei Federal $n^{\circ} 12.608$ - representa um marco na GRD do Brasil. A lei reconhece a importância da articulação entre União, estados e municípios e suas distintas políticas setoriais para GRD ${ }^{5}$.

O Plano Nacional de Saúde, a Política Nacional de Promoção de Saúde e a Política Nacional de Atenção Básica já davam ênfase à criação de estratégias para redução de quaisquer fatores que representavam riscos à saúde da populaçãa ${ }^{15-17}$.

O Programa Vigidesastres é central nesse contexto. Trata-se de um programa nacional de implementação local que se baseia na articulação do Sistema Único de Saúde (SUS) com 
as ações de promoção da saúde e redução de riscos de desastres.

Os profissionais da saúde também são apontados pelo Marco de Ação de Sendai (acordo internacional firmado na Terceira Conferência Mundial sobre a Redução do Risco de Desastres) como importantes para a redução de riscos de desastres. O documento salienta a necessidade de capacitá-los e integrá-los à GRD ${ }^{18}$.

A GRD pode ser definida como um processo em que se deseja prever, reduzir e controlar permanentemente os fatores que tornam a sociedade vulnerável aos riscos de desastres ${ }^{3}$. Na medida em que o risco existe, a intervenção possível é do tipo 'corretiva', contemplando ações que reduzem o risco e preparam a sociedade para o desastre. A intervenção 'reativa' contempla aspectos emergenciais e recuperativos; por outro lado, a gestão 'prospectiva' busca evitar que novas condições de riscos sejam criadas no território ${ }^{3}$.

Diante da complexidade dos fatores que constroem o risco, ressalta-se a necessária integração entre o setor de saúde pública e a GRD:

Um ponto essencial a ser considerando é que a primeira etapa para a gestão de risco de desastre no Setor Saúde envolve conhecer a sua própria capacidade de atuação. Para isso, é necessário estimar o risco identificando as ameaças e suas vulnerabilidades estimando riscos potenciais. Conhecendo estes potenciais riscos é possível definir um 'planejamento' onde sejam identificados os 'atores, recursos e ações' do Sistema de Saúde para atender a uma emergência ou desastre ${ }^{\mathbf{1 9 ( 1 2 1 3 )}}$ [Grifo dos autores].

\section{Uma ferramenta gerencial}

A ferramenta desenvolvida, apresentada e aplicada neste estudo surge em um contexto em que se reconhece a limitação dos planos de contingência elaborados para a área de saúde pública. Propõe-se, portanto, uma ferramenta de caráter gerencial que seja capaz de facilitar a integração entre atores, recursos e ações em uma perspectiva holística ${ }^{6,19}$.

Uma representação genérica do instrumento se apresenta na figura 2. Nela, contempla-se a articulação entre os agentes e os instrumentos jurídicos associados às suas respectivas ações de 'saúde e GRD'. As ações, por sua vez, estão contempladas em um processo da gestão e podem ou não estar vinculadas a projetos e programas (figura 2).

Figura 2. Esquema genérico do instrumento de levantamento e integração de dados para plano de ação dos serviços de saúde na Gestão de Riscos de Desastres

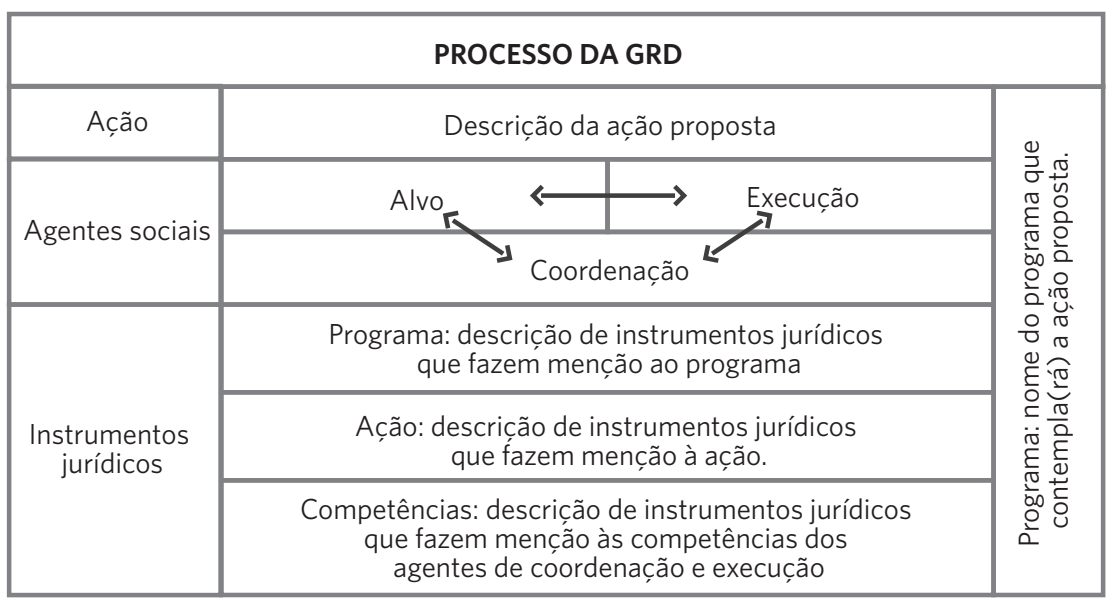


Entende-se que a elaboração de um plano de ação requer um processo de planejamento estratégico integrado (multisetorial), integral (holístico, abordando riscos e danos) e participativo. Pode-se considerá-lo como um sistema articulado por diversas dimensões: institucional, técnica, empírica, legal e operacional20.

Nos elementos da figura 2, a dimensão institucional é contemplada pelas organizações formais e informais vinculadas aos agentes sociais ${ }^{21}$. Por sua vez, a dimensão técnica e empírica é formada por agentes sociais que coordenam e executam as ações em prol de assistir e dar suporte ao agente 'alvo'.

É importante destacar que todas essas relações se dão conforme as 'regras do jogo' da dimensão legal e normativa (constituição, leis, planos, programas, projetos). As ferramentas e recursos disponíveis são definidos e aplicados em etapas operacionais (dimensão operacional).

Os processos da GRD considerados são seis: 1) geração de conhecimento; 2) prevenção e 3) mitigação de riscos; 4) preparação e 5) resposta ao desastre; e 6) recuperação. Seus conceitos podem ser encontrados no estudo de Vieira, Schmidt e Moura'.

Em relação aos agentes socais, observam-se três componentes de formação: o 'alvo', que é o agente recebedor/beneficiado da ação; o executor, que se refere a quem irá interagir diretamente com o 'alvo'; e a coordenação, que é o responsável por sistematizar, organizar e planejar a ação. É importante que esses agentes sejam bem definidos para que fique esclarecida a função de cada um dentro do processo.

Para fundamentar as ações, programas e definir competências, o instrumento destaca o panorama legal. Busca-se registrar se todo programa, ação ou competência possui embasamento e definição legal, o que permite identificar fragilidades institucionais e legais.

Reconhece-se que o estudo gera um panorama preliminar, parcial, desenvolvido em âmbito acadêmico e que necessita contar com a contribuição de todos os stakeholders envolvidos no processo de planejamento estratégico participativo. Não obstante, sua aplicação permite identificar ações e processos que até então não são contemplados pelos planos vigentes ou que não possuem uma clara definição de competências legais, sobretudo no âmbito do poder público municipal, responsável pela atenção básica de saúde.

\section{Resultados e discussão}

O resultado proveniente da aplicação do instrumento agrupa as atividades em três eixos temáticos: 1) Sensibilização, capacitação e cuidado dos profissionais da saúde; 2) Sensibilização, acolhimento e desenvolvimento de práticas educativas na comunidade; 3) Coordenação, planejamento e demais operações. A estrutura e as ações estão focadas na realidade da área de estudo, abordando as inundações e os movimentos gravitacionais de massa. A discussão foca nos possíveis papéis e na contribuição dos profissionais da saúde na GRD.

Nos quadros 1, 2 e 3 apresenta-se o resultado completo da aplicação do instrumento de sistematização de dados de dimensão institucional, técnica, empírica e legal. Embora se faça referência aos dados específicos dos quadros 1, 2 e 3, procurou-se focar a discussão em uma contribuição ampla e aprofundada sobre os possíveis 'papéis' dos profissionais da saúde na GRD.

\section{Sensibilização, capacitação e cuidado dos profissionais da saúde}

No eixo temático 1 , as ações propostas vinculam-se à capacitação e à sensibilização dos profissionais de saúde em relação aos processos de prevenção, mitigação e preparação ante os riscos de desastre naturais (quadro 1). Aborda-se o cuidado da saúde desses profissionais durante e após o evento danoso, um aspecto, por vezes, negligenciado nas políticas públicas. 
Quadro 1. Eixo temático 1 - Sensibilização, capacitação e cuidado dos profissionais de saúde. Instrumento gerencial para a gestão dos riscos de desastres, Blumenau, SC, 2019

\begin{tabular}{|c|c|c|c|c|c|c|c|c|c|}
\hline \multicolumn{10}{|c|}{ Eixo Temático: Sensibilização, capacitação e cuidados dos profissionais da saúde } \\
\hline & 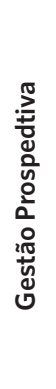 & 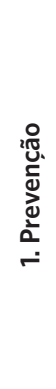 & $\begin{array}{l}\text { Agentes } \\
\text { sociais } \\
\text { Instru- } \\
\text { mentos } \\
\text { jurídicos }\end{array}$ & $\begin{array}{l}\text { 1.1 Executar, gerir, avaliar e revisar atividades de } \\
\text { capacitação de profissionais da saúde vincula- } \\
\text { das à prevenção de riscos futuros de desastres } \\
\text { naturais. } \\
\text { Profissionais de saúde Capacitador } \\
\text { Diretoria de Ações em Saúde } \\
\text { Programa: PEES } \\
\text { Ação: PNS, PNEP, PNPS, PNAB, PEES, PMS } \\
\text { Competência: PMS }\end{array}$ & 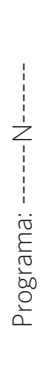 & & & & \\
\hline 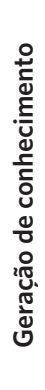 & 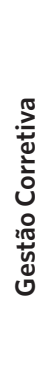 & 足 & $\begin{array}{l}\text { Agentes } \\
\text { sociais } \\
\text { Instru- } \\
\text { mentos } \\
\text { jurídicos }\end{array}$ & $\begin{array}{l}\text { 2.1 Executar, gerir, avaliar e revisar atividades } \\
\text { de capacitação de profissionais da saúde } \\
\text { vinculadas à redução de riscos de desastres } \\
\text { naturais. } \\
\text { Profissionais de saúde Capacitador } \\
\text { Diretoria de Ações em Saúde } \\
\text { Programa: PEES } \\
\text { Ação: PNS, PNEP, PNPS, PNAB, PEES, PMS } \\
\text { Competência: PMS }\end{array}$ & $\begin{array}{l}\sum_{1}^{1} \\
\vdots \\
\vdots \\
\vdots \\
0 \\
\frac{0}{0} \\
\frac{0}{00} \\
0 \\
0\end{array}$ & 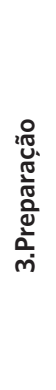 & $\begin{array}{l}\text { Agentes } \\
\text { sociais } \\
\text { Instrumen- } \\
\text { tos jurídicos }\end{array}$ & $\begin{array}{l}\text { 3.1 Executar, gerir, avaliar e revisar ativi- } \\
\text { dades de capacitação de profissionais da } \\
\text { saúde vinculadas às situações de emer- } \\
\text { gências. } \\
\text { Profissionais de saúde Capacitador } \\
\text { Diretoria de Ações em Saúde } \\
\text { Programa: PEES } \\
\text { Ação: PNS, PNEP, PNPS, PNAB, PEES, PMS } \\
\text { Competência: PMS }\end{array}$ & 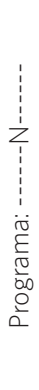 \\
\hline & 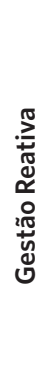 & 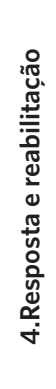 & $\begin{array}{l}\text { Agentes } \\
\text { sociais } \\
\text { Instru- } \\
\text { mentos } \\
\text { jurídicos }\end{array}$ & $\begin{array}{l}\text { 4.1 Executar, gerir, avaliar e revisar atividades } \\
\text { de acompanhamento da saúde } \\
\text { de profissionais da saúde durante a ocorrência } \\
\text { de desastres naturais. } \\
\text { Profissionais de saúde PSC, TO, MED } \\
\text { Diretoria de Vigilância em Saúde e Cerest } \\
\text { Programa: ------N------- } \\
\text { Ação: PNST, PMS } \\
\text { Competência: PNST, PMS }\end{array}$ & 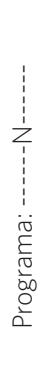 & 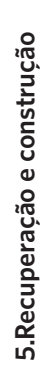 & $\begin{array}{l}\text { Agentes } \\
\text { sociais } \\
\text { Instrumen- } \\
\text { tos jurídicos }\end{array}$ & $\begin{array}{l}\text { 5.1 Executar, gerir, avaliar e revisar ativi- } \\
\text { dades de acompanhamento da saúde de } \\
\text { profissionais da saúde após a ocorrência } \\
\text { de desastres naturais. } \\
\text { Profissionais de saúde PSC, TO, MED } \\
\text { Diretoria de Vigilância em Saúde e Cerest } \\
\text { Programa: ------N------ } \\
\text { Ação: PNST, PMS } \\
\text { Competência: PMS }\end{array}$ & \\
\hline
\end{tabular}

Fonte: Elaboração própria.

Legenda (agentes sociais): Agente Comunitário de Saúde (ACS), Agente de Combate de Endemias (ACE), Técnico de Vigilância Sanitária (T. VS), Técnico de Vigilância Epidemiológica (T. VE), Técnico de Vigilância em Saúde Ambiental (T. VSA), Enfermeiro (ENF), Técnico de Enfermagem (T. ENF), Médico (MED), Psicólogo (PSC), Fisioterapeuta (FIS), Terapeuta ocupacional (TO), Centros de Referência em Saúde do Trabalhador (Cerest), Técnico de Defesa Civil (ADC), Centro de Atenção psicossocial (Caps).

Legenda (instrumentos jurídicos): Plano Nacional de Saúde (PNS), Política Nacional de Promoção da Saúde (PNPS), Política Nacional de Atenção Básica (PNAB), Política Nacional de Educação Permanente em Saúde (PNEP), Plano Estadual de Emergências em Saúde (PEES), Plano Municipal de Saúde (PMS), Política Nacional de Saúde do Trabalhador (PNST), Política Nacional de Proteção e Defesa Civil (PNPDEC), Plano de Contingência da Saúde (PCS).

Legenda (outras): ----N---- não há.

No Brasil, observam-se diversos instrumentos
jurídicos da saúde que salientam a importância
de promover a educação, a formação profissional
e a capacitação específica aos gestores e trabalha-
dores da saúde, para que sua capacidade crítica
e reflexiva seja estimulada. Essas capacitações
devem sempre levar em conta a realidade am-
biental local e condições de vida da população,
como salientada pela Organização Mundial da

Saúde (OMS) 22 e nacionalmente pela Política Nacional de Atenção Básica ${ }^{17}$.

$\mathrm{Na}$ realidade de muitos municípios, mostra-se imprescindível que sejam realizadas a sensibilização e a capacitação dos profissionais de saúde sobre o tema de risco de desastres, abordando aspectos de prevenção de risco futuro, redução de risco atual e de ações na iminência de um desastre (preparação). 
Essas ações devem ser estimuladas por uma política pública, ou seja, contempladas em um plano de ação, caso contrário, serão frágeis e dependentes de iniciativas pontuais.

No País, são vários os exemplos de ações de profissionais da saúde voltados à sensibilização da comunidade e à implementação de práticas educativas referentes à ocorrência de desastres. Em Rio Branco, no Acre, o Agente Comunitário de Saúde (ACS) e o Agente de Combate de Endemias (ACE) atuam na redução de vulnerabilidade ao orientar a comunidade sobre questões de higiene, doenças e cuidados pessoais durante os desastres ${ }^{\mathbf{2 3}}$.

Esse tipo de trabalho é possível quando há investimento na educação continuada dos profissionais de saúde sobre os riscos de desastres. Moura ${ }^{\mathbf{9}(109)}$ relata o depoimento de uma ACS de Blumenau que reconhece a necessidade:

Poderíamos receber cursos e capacitações sobre $o$ assunto para sabermos o que podermos trabalhar com a população com essa temática, para diminuir o risco e aumentar as medidas preventivas.

Do ponto de vista do dano, muitas vezes identifica-se que os próprios profissionais da área da saúde residem em área de risco e, portanto, também podem ser afetados física e emocionalmente. A Lei Federal n ${ }^{0} 8.080 / 90$ prevê a recuperação e reabilitação da saúde dos trabalhadores submetidos aos riscos e agravos advindos das condições de trabalho ${ }^{24}$, aspecto também reforçado pela OMS ${ }^{25}$.

Em Blumenau (SC), é o Centro de Referência em Saúde do Trabalhador (Cerest) que realiza promoção, prevenção, vigilância, diagnóstico, tratamento e reabilitação em saúde de todos os trabalhadores nesses eventos. Acompanhamento psicológico e ações de promoção de saúde dos próprios profissionais são importantes para que o serviço público seja humanizado.

\section{Sensibilização, acolhimento e desenvolvimento de práticas educativas junto à comunidade}

No segundo eixo temático, as ações de prevenção e mitigação dos riscos de desastres naturais, bem como de preparação ao desastre, possuem um caráter sensibilizador e educativo, isto é, visam construir práticas educativas para que a comunidade conheça seu território e perceba o risco (quadro 2). Já nos processos de resposta e recuperação, observam-se ações de cuidado à população afetada durante e após o desastre.

Quadro 2. Eixo temático 2 - Sensibilização, acolhimento e desenvolvimento de práticas educativas na comunidade. Instrumento gerencial para a gestão dos riscos de desastres, Blumenau, SC, 2019

\begin{tabular}{|c|c|c|c|c|c|}
\hline \multicolumn{6}{|c|}{ Eixo Temático: Sensibilização, acolhimento e desenvolvimento de práticas educativas junto à comunidade } \\
\hline \multirow{6}{*}{ 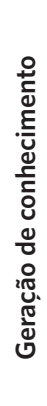 } & \multirow{6}{*}{ 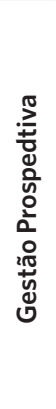 } & \multirow{6}{*}{ 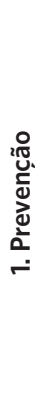 } & Ação & $\begin{array}{l}\text { 1.2 Sensibilizar a população sobre os desastres } \\
\text { naturais, com ênfase nos riscos futuros e seus } \\
\text { possíveis impactos negativos na saúde. }\end{array}$ & \multirow{6}{*}{ 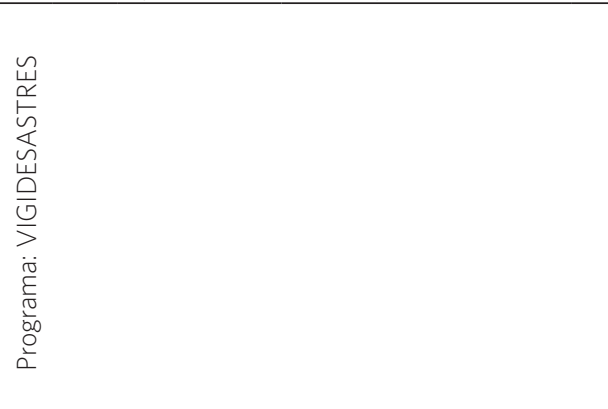 } \\
\hline & & & $\begin{array}{l}\text { Agentes } \\
\text { sociais }\end{array}$ & $\begin{array}{ll}\text { Comunidade } & \text { ACS, ACE, T. VSA, T. } \\
& V S, T . V E, A D C\end{array}$ & \\
\hline & & & & $\begin{array}{l}\text { Diretoria de Ações em Saúde, Diretoria de } \\
\text { Vigilância em Saúde, Diretoria de Defesa Civil }\end{array}$ & \\
\hline & & & \multirow{3}{*}{$\begin{array}{l}\text { Instru- } \\
\text { mentos } \\
\text { jurídicos }\end{array}$} & Programa: PEES & \\
\hline & & & & Ação: PNPS, PNAB, PEES & \\
\hline & & & & Competência: PNAB, PEES & \\
\hline
\end{tabular}


Quadro 2. (cont.)

\begin{tabular}{|c|c|c|c|c|c|c|c|c|c|}
\hline & & & & nático: Sensibilização, acolhimento e desen & & & práticas ed & Eativas junto à comunidade & \\
\hline 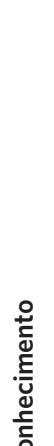 & 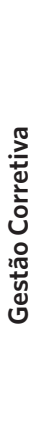 & 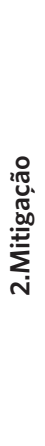 & $\begin{array}{l}\text { Agentes } \\
\text { sociais } \\
\text { Instru- } \\
\text { mentos } \\
\text { jurídicos }\end{array}$ & $\begin{array}{l}\text { 2.2 Sensibilizar a população sobre medidas bá- } \\
\text { sicas de manejo ambiental voltado à promoção } \\
\text { da saúde e à redução dos riscos de desastres } \\
\text { naturais. } \\
\text { Comunidade ACS, ACE, T. VSA, T. } \\
\qquad V S, T \text {. VE, ADC } \\
\text { Diretoria de Ações em Saúde, Diretoria de } \\
\begin{array}{r}\text { Vigilância em Saúde, Diretoria de Defesa Civil } \\
\text { Programa: PEES }\end{array} \\
\text { Ação: PNPS, PNAB, PEES } \\
\text { Competência: PNAB, PEES }\end{array}$ & 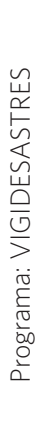 & 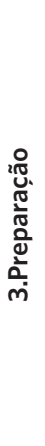 & $\begin{array}{l}\text { Instrumen- } \\
\text { tos jurídicos }\end{array}$ & $\begin{array}{l}\text { 3.2 Participar de simulados de desastre e } \\
\text { orientar a população sobre como proceder } \\
\text { na ocorrência de um desastre natural. } \\
\text { Comunidade e profis- ADC, T. VSA } \\
\text { sionais de saúde } \\
\text { Diretoria de Defesa Civil, Diretoria de } \\
\text { Vigilância em Saúde } \\
\text { Programa: PEES } \\
\text { Ação: PNPDEC } \\
\text { Competência: PNPDEC }\end{array}$ & 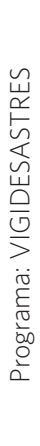 \\
\hline 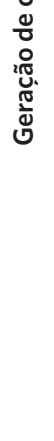 & 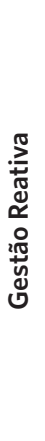 & 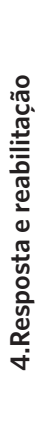 & $\begin{array}{l}\text { Instru- } \\
\text { mentos } \\
\text { jurídicos }\end{array}$ & 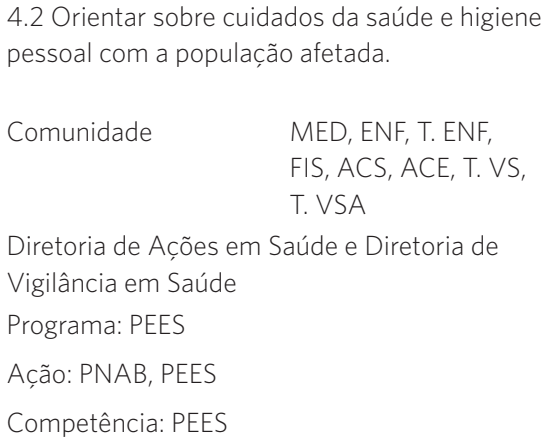 & 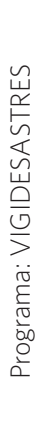 & 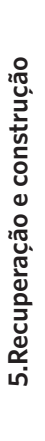 & $\begin{array}{l}\text { Instrumen- } \\
\text { tos jurídicos }\end{array}$ & $\begin{array}{l}\text { 5.2 Atender população afetada que ne- } \\
\text { cessita de acompanhamento psicossocial } \\
\text { (saúde mental). } \\
\text { Comunidade PSC, TO, MED }\end{array}$ & 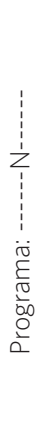 \\
\hline
\end{tabular}

Fonte: Elaboração própria.

Legenda (agentes sociais): Agente Comunitário de Saúde (ACS), Agente de Combate de Endemias (ACE), Técnico de Vigilância Sanitária (T. VS), Técnico de Vigilância Epidemiológica (T. VE), Técnico de Vigilância em Saúde Ambiental (T. VSA), Enfermeiro (ENF), Técnico de Enfermagem (T. ENF), Médico (MED), Psicólogo (PSC), Fisioterapeuta (FIS), Terapeuta ocupacional (TO), Centros de Referência em Saúde do Trabalhador (Cerest), Técnico de Defesa Civil (ADC), Centro de Atenção psicossocial (Caps).

Legenda (instrumentos jurídicos): Plano Nacional de Saúde (PNS), Política Nacional de Promoção da Saúde (PNPS), Política Nacional de Atenção Básica (PNAB), Política Nacional de Educação Permanente em Saúde (PNEP), Plano Estadual de Emergências em Saúde (PEES), Plano Municipal de Saúde (PMS), Política Nacional de Saúde do Trabalhador (PNST), Política Nacional de Proteção e Defesa Civil (PNPDEC), Plano de Contingência da Saúde (PCS).

Legenda (outras): ----N---- não há.

Atualmente, nenhuma das ações indicadas é contemplada pela legislação municipal, ainda que sejam contempladas em instrumentos jurídicos federais e estaduais por meio do Programa Vigidesastres (quadro 2). Esse programa insere-se na Vigilância em Saúde Ambiental (VSA) e objetiva realizar ações para reduzir, de maneira integral, a vulnerabilidade da população e dos profissionais de saúde. Assim, o caráter integrador da VSA aponta:

[...] especificidades próprias e, ao mesmo tempo, interfaces com a vigilância sanitária, a vigilância epidemiológica, a saúde do trabalhador, os laboratórios de saúde pública e o saneamento ambiental, como áreas de intervenção organizadas no âmbito do SUS, e, ainda, como já dito anteriormente, que perpassam por muitos outros Ministérios 26(30).

Em Blumenau (SC), as ações da VSA vinculadas à prevenção e à mitigação de riscos não são apresentadas em nenhuma política ou plano. Observa-se essa visão integrada no plano estadual e federal, mas ainda de forma generalista 9 . Afinal, é no município que a gestão efetivamente se consolida e cria mecanismos práticos para a sua implementação.

No contexto de Blumenau (SC), os resultados também apontam a importância 
de integrar a Diretoria de Vigilância em Saúde (Vigilâncias Ambiental, Sanitária e Epidemiológica) e a Diretoria de Ações de Saúde (forte ligação com a atenção básica). Juntas elas irão coordenar ações importantes de saúde ambiental, controle de doenças e promoção da saúde ${ }^{27}$. Por essa razão, essas duas diretorias possuem uma presença significativa nas ações do Eixo 2 (quadro 2). A nova Política Nacional de Atenção Básica ${ }^{17(19)}$ (item 4.2.6) afirma também que a:

\section{Atenção básica e vigilância em saúde devem se unir para a adequada identificação de proble- mas de saúde nos territórios e planejamento de estratégias de intervenção clínica e sanitária mais efetivas e eficazes $[\ldots]^{\mathbf{1 7}(19)}$.}

Essa política orienta que as atividades específicas dos agentes de saúde (ACS e ACE) devem ser integradas, dando embasamento jurídico para que eles atuem em conjunto nas ações propostas (quadro 2).

Em consonância, o Plano Estadual de Emergência de SC declara que:

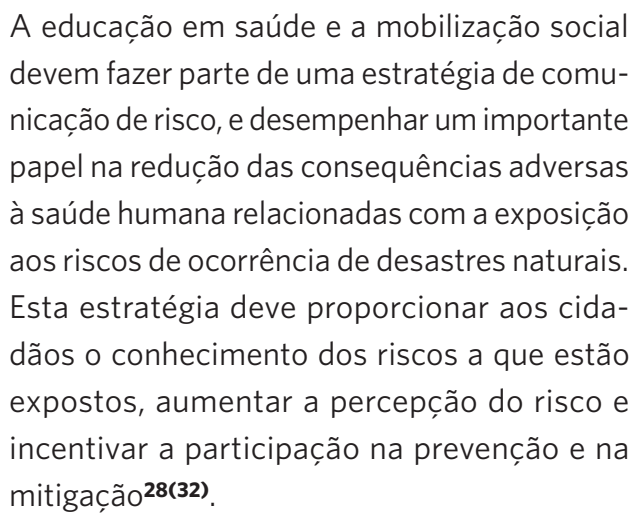

Os profissionais da saúde citados como agentes de execução de ações de sensibilização podem construir com a população práticas sensibilizadoras de manejo ambiental e identificação de sinais de perigo associado aos fenômenos naturais e tecnológicos. Medidas simples também são sugeridas pela OMS ${ }^{29(2)}$, como: “[...]Prevenir infecções por meio de práticas educativas associados ao fornecimento de instalações e materiais de higiene pessoal" [tradução dos autores].

No que concerne à realização dos simulados de emergência, o Plano Estadual de Emergências de SC enfatiza que o Programa Vigidesastres também pode realizar ações vinculadas à preparação e alerta na Defesa Civil28. Apesar da Defesa Civil de Blumenau (SC) realizar simulados de desastres associados à movimentação gravitacional de massa, não há registros de participação formal dos profissionais de saúde?

No que diz respeito às ações vinculadas ao cuidado com a saúde da comunidade durante e após a ocorrência do desastre, estas podem ser realizadas por diversos agentes de saúde, incluindo médicos, enfermeiros, técnicos de enfermagem, fisioterapeutas, psicólogos, terapeutas ocupacionais, técnicos de vigilância ambiental e sanitária, agentes comunitários de saúde, agentes de combate a endemias. Todos podem ser inseridos em uma política de atenção, recuperação, reabilitação e acompanhamento psicossocial.

Nesse sentido, destaca-se o Centro de Atenção Psicossocial (Caps) no processo de recuperação da população de Blumenau (SC). Trata-se de um serviço capaz de articular ações com as equipes de atenção básica visando atender integralmente a comunidade afetada pelos desastres. É essencial que esse acompanhamento seja longitudinal e que inclua, além dos aspectos psicossomáticos, os sociais e ambientais; questões estas que podem ser abordadas de maneira intersetorial (recursos comunitários, instituições e organizações públicas e privadas) pelas equipes multiprofissionais e interdisciplinares do Caps.

\section{Coordenação, planejamento e demais operações}

Neste eixo temático (quadro 3), as ações que se vinculam ao processo de mitigação visam identificar vulnerabilidades e construções impróprias, sistematizar dados sobre os riscos de desastres e incentivar a participação social. 
Quadro 3. Eixo temático 3 - Coordenação, planejamento e demais operações. Instrumento gerencial para a gestão dos riscos de desastres, Blumenau, SC, 2019

Eixo Temático: Coordenação, planejamento e demais operações.

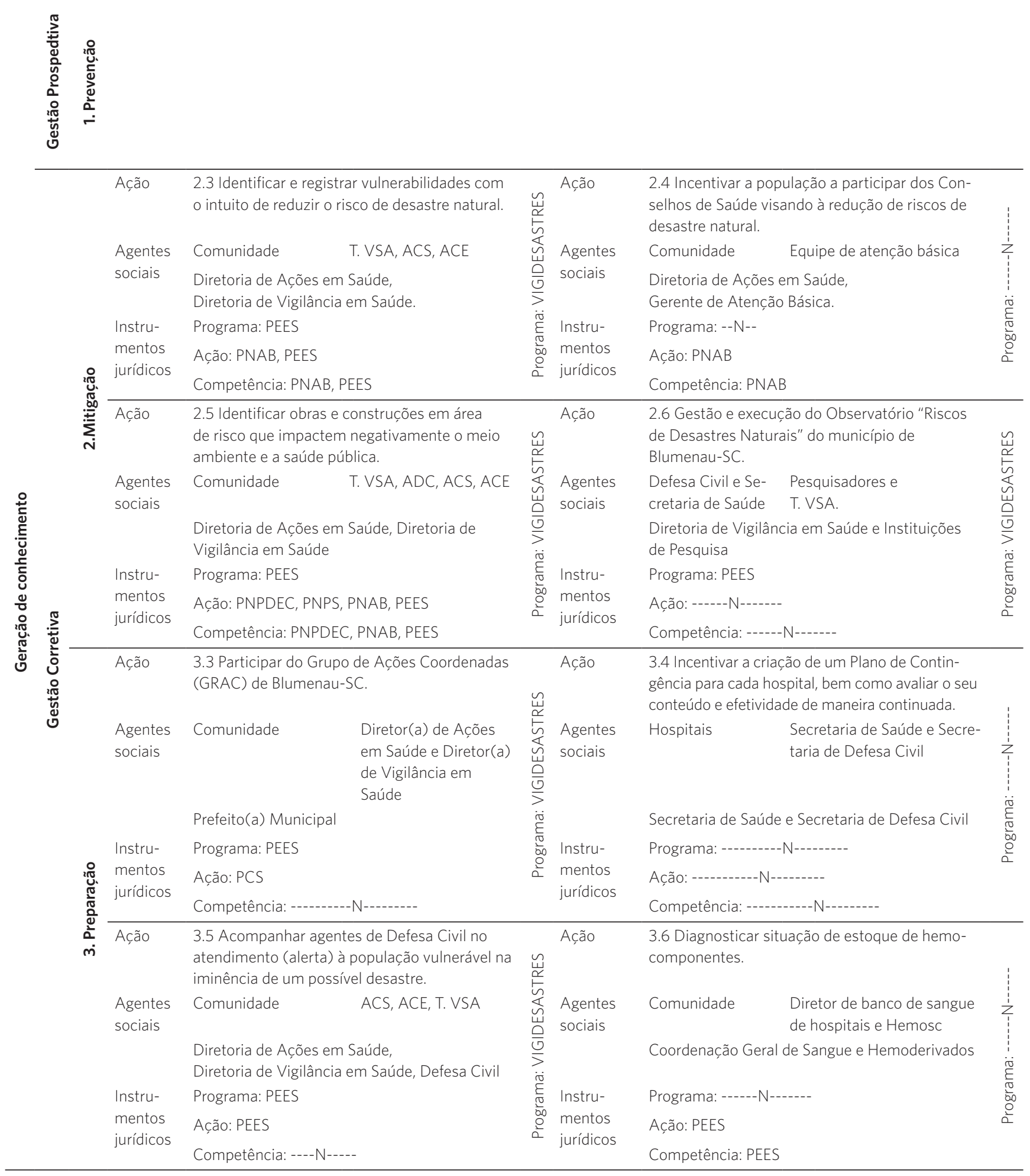


Eixo Temático: Coordenação, planejamento e demais operações.

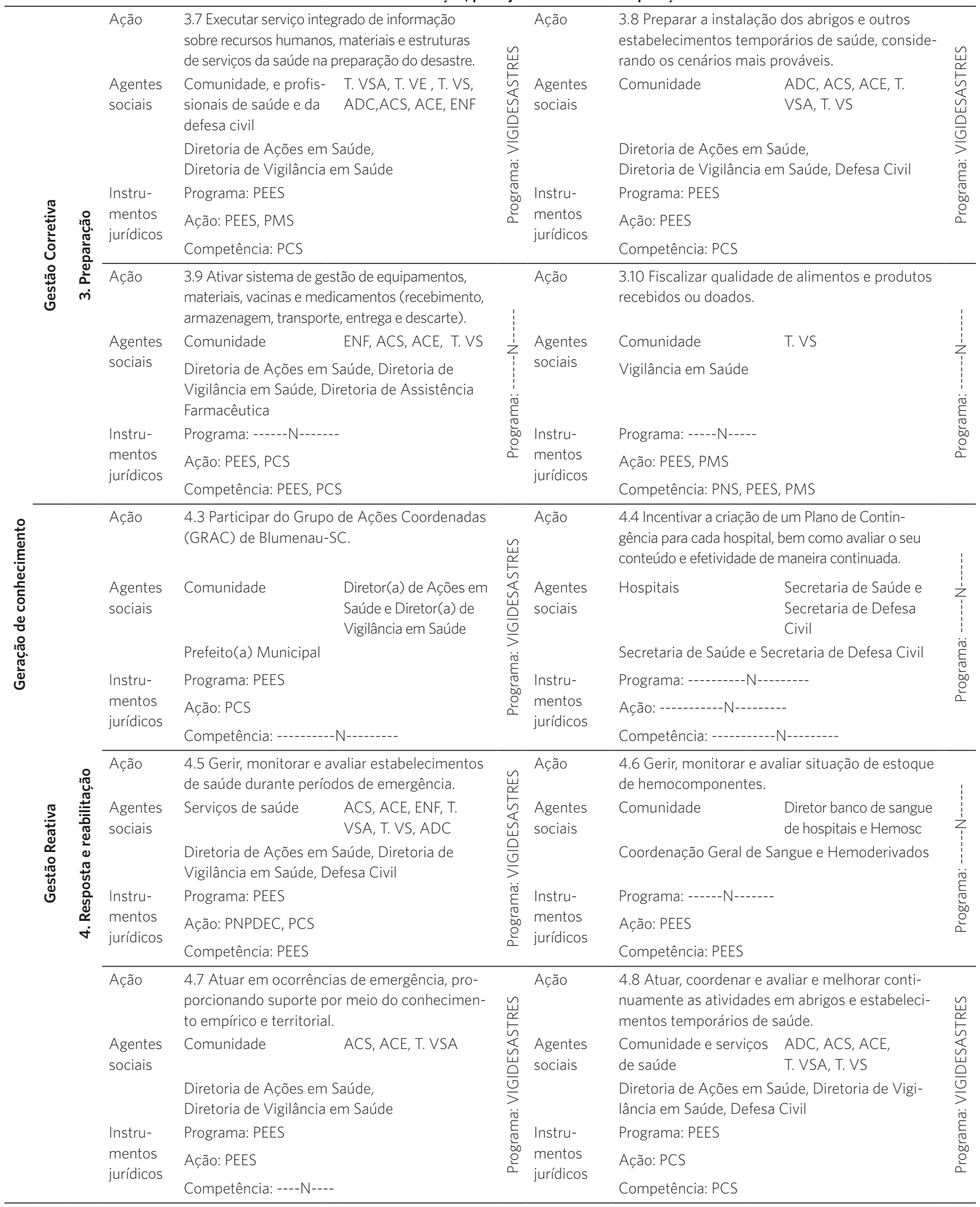


Quadro 3. (cont.)

\begin{tabular}{|c|c|c|c|c|c|c|c|c|}
\hline & & & & Eixo Temático: Coordenação, pl & iejan & nento e de & is operações. & \\
\hline & & & Ação & $\begin{array}{l}4.9 \text { Atuar, coordenar e avaliar e melhorar con- } \\
\text { tinuamente sistema de gestão de materiais e } \\
\text { medicamentos. }\end{array}$ & & Ação & $\begin{array}{l}\text { 4.10 Promover boas práticas para o acesso uni- } \\
\text { versal à água potável. }\end{array}$ & \\
\hline & & & Agentes & Comunidade $\quad$ ENF, ACS, T. VS & $\stackrel{1}{z}$ & Agentes & 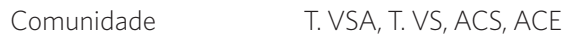 & \\
\hline & & 窎 & sociais & $\begin{array}{l}\text { Diretoria de Ações em Saúde, } \\
\text { Diretoria de Vigilância em Saúde, } \\
\text { Diretoria de Assistência Farmacêutica }\end{array}$ & 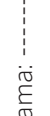 & sociais & $\begin{array}{l}\text { Diretoria de Ações em Saúde, } \\
\text { Diretoria de Vigilância em Saúde }\end{array}$ & 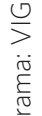 \\
\hline & & 喜 & Instru- & Programa: ------N------ & $\frac{10}{0.0}$ & Instru- & Programa: PEES, PMS & \\
\hline & & 岕 & mentos & Ação: PCS & & mentos & Ação: PEES, PCS & \\
\hline & & 焉 & & Competência: PCS & & & Competência: PEES, PCS & \\
\hline 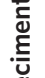 &.$\stackrel{2}{\Sigma}$ & 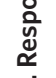 & Ação & $\begin{array}{l}4.11 \text { Promover medidas de prevenção a } \\
\text { zoonoses. }\end{array}$ & & & & \\
\hline$\frac{\mathscr{c}}{5}$ & 苞 & $\dot{\nabla}$ & Agentes & Comunidade $\quad$ T.VS & $\sum_{1}^{1}$ & & & \\
\hline$\underset{u}{\tilde{\pi}}$ & 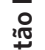 & & sociais & Diretoria de Vigilância em Saúde & & & & \\
\hline 20 & $\stackrel{\mathscr{U}}{\mathrm{U}}$ & & Instru- & Programa:------N------- & $\ddot{\ddot{E}}$ & & & \\
\hline$\frac{\pi}{20}$ & & & mentos & Ação: PEES, PCS, PMS & $\frac{5}{00}$ & & & \\
\hline & & & & Competência: PEES, PCS, PMS & & & & \\
\hline & & : & Ação & $\begin{array}{l}\text { 5.3 Desenvolver Plano de Recuperação em } \\
\text { Saúde Pós- Desastre Natural (PRS-PDN) }\end{array}$ & 㟧 & & & \\
\hline & & 蒙 & Agentes & Comunidade $\quad$ ADC, T. VSA & 㐫 & & & \\
\hline & & $\begin{array}{l}\frac{0}{2} \\
0 \\
0\end{array}$ & & Diretoria de Vigilância em Saúde & $\frac{\mathrm{O}}{\mathrm{U}}$ & & & \\
\hline & & 莺 & Instru- & Programa: PEES & $\underset{i \sigma}{>}$ & & & \\
\hline & & $\frac{0}{3}$ & jurídicos & Ação: ----------N--------- & $\frac{\sqrt{0}}{0.0}$ & & & \\
\hline & & $\frac{\mathscr{\alpha}}{\dot{H}}$ & & Competência: ----N---- & c & & & \\
\hline
\end{tabular}

Fonte: Elaboração própria.

Legenda (agentes sociais): Agente Comunitário de Saúde (ACS), Agente de Combate de Endemias (ACE), Técnico de Vigilância Sanitária (T. VS), Técnico de Vigilância Epidemiológica (T. VE), Técnico de Vigilância em Saúde Ambiental (T. VSA), Enfermeiro (ENF), Técnico de Enfermagem (T. ENF), Médico (MED), Psicólogo (PSC), Fisioterapeuta (FIS), Terapeuta ocupacional (TO), Centros de Referência em Saúde do Trabalhador (Cerest), Técnico de Defesa Civil (ADC), Centro de Atenção psicossocial (Caps).

Legenda (instrumentos jurídicos): Plano Nacional de Saúde (PNS), Política Nacional de Promoção da Saúde (PNPS), Política Nacional de Atenção Básica (PNAB), Política Nacional de Educação Permanente em Saúde (PNEP), Plano Estadual de Emergências em Saúde (PEES), Plano Municipal de Saúde (PMS), Política Nacional de Saúde do Trabalhador (PNST), Política Nacional de Proteção e Defesa Civil (PNPDEC), Plano de Contingência da Saúde (PCS).

Legenda (outras): ----N---- não há.

A Política Nacional de Atenção Básica define como atribuição das equipes de atenção básica a participação do processo de territorialização e mapeamento da área de atuação, identificando grupos, famílias e indivíduos expostos a riscos e vulnerabilidades ${ }^{17}$. Dados coletados formal ou informalmente pelos ACS, pelos ACE e pelo Técnico de Vigilância em Saúde Ambiental (TVSA) podem ser considerados nas políticas de GRD (quadro 3).

Radicchi e Lemos ${ }^{30}$ ressaltam a importância de o setor da saúde agir de forma articulada e mobilizadora, influenciando na sensibilização e argumentação da sociedade e nas tomadas de decisões do poder público. Sobral et al.31 também salientam a necessidade da articulação entre Vigilância e Atenção Básica de saúde por meio de ações vinculadas à GRD. A Portaria do Ministério da Saúde n ${ }^{\circ} 1.378$, de julho de 2013, afirma a necessidade de articulação entre a vigilância em saúde e a atenção básica de saúde com ações que visem ao controle de riscos à saúde, envolvendo, portanto, a integração nas ações vinculadas aos desastres ${ }^{32}$. 
Os ACS, os ACE e o TVSA podem auxiliar na identificação de condições ambientais perigosas e construções impróprias tendo em vista seu envolvimento com a comunidade, seu conhecimento empírico do território e sua vivência local. Todos esses fatores podem apoiar um trabalho intersetorial com técnicos de Defesa Civil, que possuem o conhecimento técnico, específico, mas 'aparecem' com menos frequência nas localidades ${ }^{33}$.

Outra ação proposta e descrita (quadro 3) se refere à criação de um Observatório sobre os Riscos de Desastres Naturais. Essa instituição ainda não existe no município e poderia coordenar, sistematizar e divulgar dados e relatórios técnico-científicos relacionados com os temas saúde ambiental, desastres e epidemiologia. Os estudos podem subsidiar as tomadas de decisões e a formulação de políticas públicas.

De igual modo, é importante que os profissionais de saúde incentivem a participação social nos conselhos de saúde locais e municipal. As ações construídas coletivamente tendem a filtrar melhor o que é prioridade para a comunidade. O quadro 3 aponta também para a importância da intersetorialidade.

Nas ações relacionadas com o processo de preparação, são apresentadas atividades de gestão de equipamentos, medicamentos, recursos, estruturas de saúde, hemocomponentes e insumos. Em Blumenau (SC), essas ações são previstas no plano de contingência elaborado e coordenado pela Gerência de Vigilância Sanitária (VS) ${ }^{\mathbf{1 4}}$. Contudo, observa-se que as competências e ações são pouco detalhadas. Sugere-se que as equipe da atenção básica trabalhem integrada à Gerência da VS nas ações vinculadas à preparação de equipamentos, medicamentos e estruturas de saúde para os desastres; medida que poderia estar vinculada à implementação do Programa Vigidesastres ${ }^{28}$.

A defesa civil de Blumenau (SC) tem centralizado as ações de alerta no município'. Visando uma maior articulação entre os setores, propõe-se que o alerta possa ser realizado em parceria com os profissionais de saúde. Eles possuem forte conhecimento territorial e vínculo comunitário, aspectos importantes para uma operação complexa, com especificidades particulares em cada região.

A proposta de elaboração de plano de contingência específico para os hospitais sugere que ele seja construído com a participação dos próprios estabelecimentos, técnicos da saúde e da defesa civil. Deve-se contemplar a constante avaliação de seu conteúdo após cada evento, levantando e discutindo fragilidades e potencialidades.

Ademais, devido às situações de desastres naturais gerarem danos humanos e alta demanda de suprimento de sangue, espera-se que o estoque de hemocomponentes esteja preparado previamente para que seja possível atender a todas as solicitações requeridas. Nesse caso, o Sistema Estadual de Hematologia e Hemoterapia, o Centro de Hematologia e Hemoterapia (Hemosc) do município e a defesa civil poderiam se corresponsabilizar pelo planejamento e operação da gestão de hemocomponentes ${ }^{\mathbf{2 8}}$.

No processo de resposta ao desastre, observa-se que as ações apresentadas se vinculam à atuação dos profissionais de saúde nas situações de emergência. São ações que abordam a avaliação e o monitoramento dos estabelecimentos de saúde, a realização de melhorias da gestão dos estoques de hemocomponentes, dos medicamentos e nas atividades em abrigos. Além disso, há ações relacionadas com o uso adequado da água e promoção de medidas para o controle de zoonoses.

Faz-se necessário que sejam constantemente avaliados e monitorados os danos causados nos estabelecimentos e serviços de saúde. No plano de contingência de Blumenau (SC), consta que uma das funções da VS é realizar a avaliação dos serviços de saúde. As equipes de atenção básica também podem contribuir para ação, juntamente com a defesa civil, estimando a magnitude de possíveis danos aos estabelecimentos ${ }^{\mathbf{2 8}}$.

Um exemplo da atuação da atenção à saúde nesse processo é descrito no plano de 
contingência da saúde do estado do Rio Grande do Sul. As unidades de saúde dos municípios gaúchos realizam um relatório contendo informações referentes aos impactos negativos ocorridos nas estruturas e operações ${ }^{34}$.

O plano de contingência da saúde de Blumenau (SC) já prevê a avaliação das atividades em abrigos, estabelecimentos temporários de saúde e dos sistemas de gestão de materiais, medicamentos e hemocomponentes. Todavia, não se sabe se efetivamente esse procedimento está implementado?.

Os técnicos de VS de Blumenau (SC) realizam medidas para o controle de zoonoses em período de desastres e contemplam o programa Vigiágua, informando a população sobre o uso adequado da água e sobre os cuidados para que se garanta sua potabilidade ${ }^{\mathbf{1 4}}$. A respeito da promoção dessas boas práticas, propõe-se que também as equipe de atenção básica ofereçam cursos sobre a temática para as comunidades mais vulneráveis.

Por fim, articulado ao processo de recuperação, salienta-se a necessidade de elaboração de um plano de recuperação em saúde no pós-desastre. Nele, podem constar ações vinculadas à execução, à gestão, à avaliação e à revisão de atividades de acompanhamento em longo prazo da saúde da comunidade afetada, bem como dos profissionais da saúde.

Contemplar-se-ia no documento a gestão e a reconstrução dos estabelecimentos de saúde prejudicados e as metas de remanejamento de unidades de saúde que se localizam em locais de risco. As instalações de saúde devem atuar de forma a permanecer acessíveis e em operação com sua capacidade máxima durante e após o desastre ${ }^{4}$.

Tal plano de recuperação é inexistente no município de Blumenau (SC), e não foram encontradas outras iniciativas semelhantes no Brasil. Medidas que evitem a criação de novos riscos também devem ser consideradas, portanto:

[...] não se trata, por exemplo, de recuperar unidades básicas de saúde em áreas de alta suscetibilidade aos movimentos gravitacional de massa ou inundação, mas sim reconstruí- las de uma maneira mais segura e acessível. Isto é, não se trata de reproduzir as condições precárias e 'perigosas' que havia no cenário de pré-impacto sob a ideia de uma 'resiliência' que na verdade se constitui em uma 'persistência' ao risco $\mathbf{4 ( 9 1 )}$

\section{Considerações finais}

O objetivo do estudo foi apresentar um instrumento técnico e sua aplicação na formulação de um Plano de Ação dos Serviços da Saúde na GRD. Embora, a aplicação tenha sido parcial e necessite de um processo participativo da sociedade, os resultados indicaram que os profissionais da saúde podem contribuir para a GRD de uma maneira ampla e integrada, assim como se prevê na legislação federal e estadual catarinense.

O plano de ação, diferentemente de um plano de contingência, caracteriza-se por abranger todos os processos que envolvem a GRD, sendo eles a geração de conhecimento, prevenção, mitigação, preparação, resposta e recuperação. A partir de então, sistematizaram-se as informações e articularam-se os principais agentes, programas e instrumentos jurídicos associados à saúde pública municipal e a GRD.

Os resultados apontaram as potencialidades e as fragilidades atuais dessas relações no município de Blumenau (SC). Observou-se que algumas ações e processos de GRD não são contemplados pelo plano municipal de saúde e pelo plano de contingência da saúde. Nesses planos, não há uma clara definição de competências legais para algumas ações importantes, entre elas, aquelas vinculadas ao Programa Vigidesastres.

Reconhece-se que os resultados levantados e discutidos não esgotam o tema, exigindo do processo de formulação do plano de ação uma intensa participação da sociedade. Contudo, os aspectos aqui levantados podem nortear 
novas iniciativas e avanços para integrar os profissionais da saúde pública à GRD nas diversas regiões do País.

Espera-se que, por meio das propostas discutidas e do instrumento técnico desenvolvido neste artigo, o processo de formulação de Plano de Ação dos Serviços da Saúde seja consolidado no âmbito da GRD. A implantação dos planos nos municípios dependerá do fortalecimento do SUS e da integração de seus profissionais, colocando-os como importantes protagonistas na redução dos riscos de desastres.

\section{Colaboradores}

Moura SRS (0000-0002-4466-5341)*, Moura JMBM (0000-0002-4847-3160)* e Vieira R (0000-0002-4399-8398)* contribuíram igualmente para a elaboração do manuscrito.

\section{Referências}

1. Vieira R, Schmidt G, Moura JMBM. Política pública urbana de gestão de riscos de desastres naturais em Blumenau-SC: processos e ações. Amb. Soc. 2019; (22):e01182.

2. Avila MRR, Mattedi MA. Disaster and territory: the production of vulnerability to disasters in the city of Blumenau/SC. Rev. Bra. Gest. Urb. 2017; 9(2):187-202.

3. Narváez L, Lavell A, Ortega GP. La gestión del riesgo de desastres: un enfoque basado en procesos. San Isidro: Secretaría General de la Comunidad Andina; 2009.

4. Freitas CM, Rocha V. Agentes locais em desastres naturais: defesa civil e saúde na redução de riscos. Rio de Janeiro: Fiocruz; 2014

5. Brasil. Lei $n^{\circ} 12.608$, de 10 de abril de 2012. Institui a política nacional de proteção e Defesa Civil-PNPDEC; dispõe sobre o sistema nacional de proteção e Defesa Civil-sinpdec eo conselho nacional de proteção e Defesa Civil-conpdec; autoriza a criação de sistema de informações e monitoramento de desastres. Diário Oficial da União. 11 Abr 2012.

6. Moura JMBM, Moura SRS, Vieira R. Bases conceituais para uma política de saúde pública integrada à gestão de riscos de desastres naturais. In: Ribeiro EAW, Miranda M, Castro RC. Mudanças ambientais, desastres e vulnerabilidade social. Blumenau: IFC; 2019. p. 87-94.

7. Moura SRS, Vieira R. Integração dos serviços de saúde pública na gestão de risco de desastres: oportunidades na atuação dos agentes comunitários de saúde. Sociedade e Território. 2019; 31(2):49-72.
*Orcid (Open Researcher and Contributor ID). 
8. Instituto Brasileiro de Geografia e Estatística [internet]. Panorama das Cidades. Blumenau: IBGE; 2019. [acesso em 2019 set 20]. Disponível em: https://cidades.ibge.gov.br/brasil/sc/blumenau/panorama.

9. Moura SRS. A política pública de saúde pública municipal no contexto da gestão de riscos de desastres naturais: o caso de Blumenau-SC [dissertação]. Blumenau: Fundação Universidade Regional de Blumenau; 2019. 167 p.

10. Siebert C. O (des)controle urbano. In: Frank B, Sevegnani L. Desastres de 2008 no Vale do Itajaí. Água, gente e política. Blumenau: Agência de Água do Vale do Itajaí; 2009. p. 110-127.

11. Sevegnani L, Frank B, Negredo JC, et al. Gente socorrendo gente. In: Frank B, Sevegnani L. Desastres de 2008 no Vale do Itajaí. Água, gente e política. Blumenau: Agência de Água do Vale do Itajaí; 2009.

12. Blumenau. Registro de inundações da defesa civil [internet]. 2019. [acesso em 2019 set 2]. Disponível em: http://www.blumenau.sc.gov.br/secretarias/secretaria-de-defesa-do-cidadao/sedeci/defesa-civil-sensibiliza-populacao-sobre-simulado-de-deslizamento50.

13. Takeshita BT, Rowe A, Nascimento FA, et al. Can Natural Disasters Increase the Risk of Stroke? J Bras Neurocirurg. 2016; 27 (1):39-45

14. Blumenau. Plano de ações da Vigilância Sanitária frente a situações de emergência ou calamidade pública. Blumenau: Prefeitura; 2018.

15. Brasil. Ministério da Saúde. Plano Nacional da Saúde [internet]. 2016. [acesso em 2017 maio 13]. Disponível em: http://conselho.saude.gov.br/ultimas_noticias/2016/docs/PlanoNacionalSaude_2016_2019.pdf.

16. Brasil. Ministério da Saúde. Política Nacional de Promoção de Saúde [internet]. 2010. [acesso em 2017 maio 13]. Disponível em: http://bvsms.saude.gov.br/bvs/ publicacoes/politica_nacional_promocao_saude_3ed. pdf.
17. Brasil. Ministério da Saúde. Portaria n ${ }^{\circ} 2436$ de 21 de setembro de 2017. Aprova a Política Nacional de Atenção Básica, estabelecendo a revisão de diretrizes para a organização da Atenção Básica, no âmbito do Sistema Único de Saúde (SUS). Diário Oficial da União. 21 Set 2017.

18. United Nations Office for Disaster Risk Reduction. Guidelines for National Platforms for Disaster Risk Reduction [internet]. 2015. [acesso em 2018 abr 11]. Disponível em: https://www.unisdr.org/we/inform/ publications/601.

19. Silva EL, Gurgel HC, Freitas CM. Gestão de risco de desastres: cenários e potenciais impactos. In: Anais do $8^{\circ}$ Simpósio Nacional de Geografia em Saúde; 2017 Junho 26; Dourados. Mato Grosso do Sul: UFGD; 2017. p. 1201-1218.

20. Phillip Junior A. Sistemas de meio ambiente: evolução em termos institucionais, legais, técnicos e operacionais. In: NISAM 2004 - Ciclo de Conferências sobre Política e Gestão Ambiental; 17 out 2004; Florianópolis. Santa Catarina: NISAM USP; 2004. p. 1-41.

21. Oliveira NG, Martins CHB. Dimensão institucional da sustentabilidade: gestão ambiental em municípios gaúchos. Ind. Econ. FEE. 2009; 37(1):65-85

22. Organização Mundial da Saúde. Strengthening national health emergency and disaster management capacities and resilience of health systems [internet]. 2011. [acesso em 2019 dez 20]. Disponível em: http:// apps.who.int/gb/ebwha/pdf_files/EB128/B128_R10-en.pdf?ua=1.

23. Rio Branco. Prefeitura, Vigilância em Saúde nos Desastres. A experiência de Rio Branco [internet]. Rio Branco: Prefeitura; 2010. [acesso em 2018 fev 4]. Disponível em: https://www.arca.fiocruz.br/handle/ icict/6867? mode=full.

24. Brasil. Lei no 8.080 , de 19 de setembro de 1990. Dispõe sobre as condições para a promoção, proteção e recuperação da saúde, a organização e o funcionamento dos serviços correspondentes e dá outras 
providências. Diário Oficial da União. 20 Set 1990.

25. Organização Mundial da Saúde. Safe hospitals: prepared for emergencies and disasters [internet]. 2017. [acesso em 2019 dez 20]. Disponível em: https://www. who.int/hac/techguidance/preparedness/risk-management-safe-hospitals-december2017.pdf?ua=1.

26. Brasil. Ministério da Saúde. Subsídios para construção da Política Nacional de Saúde Ambiental. Brasília, DF: Conselho Nacional de Saúde; 2007.

27. Santa Catarina. Plano Estadual de Saúde. Florianópolis: Secretaria Estadual da Saúde; 2016.

28. Santa Catarina. Plano Estadual de Emergência em Saúde de Santa Catarina. Florianópolis: Secretaria Estadual da Saúde; 2016.

29. Organização Mundial da Saúde. Water, sanitation and hygiene [internet]. 2017. [acesso em $2019 \mathrm{dez}$ 20]. Disponível em: https://www.who.int/hac/techguidance/preparedness/risk-management-wash-december2017.pdf?ua=1.

30. Radicchi ALA, Lemos AF. Saúde ambiental [internet]. 2009. [acesso em 2018 dez 21]. Disponível em: https:// ares. unasus. gov. br/acervo/handle/ARES/1826.
31. Sobral A, Freitas CM, Andrade EV, et al. Desastres naturais-sistemas de informação e vigilância: uma revisão da literatura. Epidemiol. Serv. Saúde. 2010; 19(4):389-402.

32. Brasil. Ministério da Saúde. Portaria ${ }^{\circ} 1.378$ de 9 de julho de 2013. Regulamenta as responsabilidades e define diretrizes para execução e financiamento das ações de Vigilância em Saúde pela União, Estados, Distrito Federal e Municípios, relativos ao Sistema Nacional de Vigilância em Saúde e Sistema Nacional de Vigilância Sanitária. Diário Oficial da União. $10 \mathrm{Jul} 2013$.

33. Universidade Aberta do Sistema Único de Saúde. Gestão local de desastres naturais para a atenção básica. Brasília, DF: UNASUS; 2016.

34. Rio Grande do Sul. Plano de contingência da Saúde para Desastres [internet], 2014. [acesso em 2017 set 27]. Disponível em: http://www.cevs.rs.gov.br/upload/arquivos/201706/07121355-plano-de-contingencia-estadual-da-saude-para-desastres.pdf.

Recebido em 14/04/2019

Aprovado em 01/10/2019

Conflito de interesses: inexistente

Suporte financeiro: o presente trabalho foi realizado com apoio da Coordenação de Aperfeiçoamento de Pessoal de Nível Superior Brasil (Capes) - Código de Financiamento 001 